\title{
Rechtsprechung
}

\section{Zur Nutzung rechtswidrig erstellten Filmmaterials im Rahmen einer Film- dokumentation}

Landgericht Berlin, Urteil vom 10. Mai 2012 - 16 O 199/11

1. Wenn auf fremden Grund ohne Zustimmung des Grundstückseigentümer gefilmt wird, dann darf dieses Filmmaterial nicht im Rahmen einer Dokumentation verwendet werden (Umsetzung von BGH GRUR 2011, 323 Preußische Schlösser und Gärten).

2. Ein Regisseur kann sich nicht auf die Kunstfreiheit berufen, wenn er sich die erkennbar eigenmächtige Inanspruchnahme fremden Eigentums zunutze macht, indem er auf der bildlichen Dokumentation dieser Handlungen aufbaut und diese unverändert und kommentarlos wiedergibt. (Leitsätze des Einsenders*)

\section{Tatbestand}

- Die Klägerin betreibt in Berlin den öffentlichen Personennahverkehr mit Bussen und U-Bahnen. Sie ist eine Anstalt öffentlichen Rechts, deren Gewährträger das Land Berlin ist. Durch das Eigenbetriebsreformgesetz vom 09. Juli 1993 wurde sie mit Wirkung zum 01. Januar 1994 Gesamtrechtsnachfolgerin des Sondervermögens „Eigenbetrieb Berliner Verkehrsbetriebe (BVG)”. Sie ist im Grundbuch u.a. wie folgt als Eigentümerin eingetragen: a) im Grundbuch des Amtsgerichts Schöneberg von Dahlem BI. 16 als Eigentümerin der Verkehrsflächen für die U-Bahnhöfe Oskar-Helene-Heim, Thielplatz, Dahlem-Dorf und Podbielskiallee, sowie dazwischen liegender Verkehrsflächen, b) im Grundbuch des Amtsgerichts Schöneberg von Zehlendorf BI. $17 \mathrm{~W}$ als Eigentümerin der Verkehrsflächen für die U-Bahnhöfe der U-Bahnhöfe Krumme Lanke und Onkel Toms Hütte nebst dazwischen liegender Verkehrsflächen. Die Einzelheiten sind den Anlagen K 9 und K 10 zu entnehmen.

Die Verkehrsanlagen der Klägerin, insbesondere die U-Bahnzüge, werden vielfach gegen ihren Willen mit Graffitis versehen. Mit diesem Thema beschäftigt sich der Film „Unlike U”, in dem die Graffitisprayer ihre Sicht der Dinge darlegen dürfen. Dazu werden Aufnahmen von U-Bahnwaggons, Bahnhöfen und sonstigen Betriebsanlagen der Klägerin gezeigt, sowie Sequenzen, in denen Unbekannte in die Tunnelanlagen eindringen und Züge bemalen.

Der Beklagte zu 1) ist zusammen mit B. B. Produzent und Regisseur des Films. Die vom Beklagten zu 1) betriebene, unter www.b.com abrufbare Internetseite enthält einen Link zu der unter www.m.com abrufbaren Internetseite der Beklagten zu 2), über die sie DVDs des Films zum Kauf anbot. Die DVDs enthielten neben dem eigentlichen Film zusätzliches Bonusmaterial gleichen Inhalts. Die Einzelheiten der Darstellung sind der Klageschrift zu entnehmen. Im Bonus-Material werden im Kapitel „Trains on line“ u.a. Aufnahmen einer routinemäßigen Reinigung eines unbemalten U-Bahnzuges gezeigt, die Mitarbeiter des Be- klagten zu 1) am 11. März 2010 in der Betriebwerkstatt Friedrichsfelde machten. Dem vorangegangen war eine mit E-Mail vom 26. Oktober 2009 an die Pressestelle gerichtete Anfrage des Produktionsassistenten T. , in der er sich als Student des Journalismus vorstellte, der für ein Jugendmagazin einen Beitrag zum Thema Graffiti erstellen wolle und dazu um eine Drehgenehmigung für Filmaufnahmen über die Reinigung eines bemalten Zuges nachsuchte. Im Anschluss daran kam es am 24. November 2009 zu einer Besprechung, deren Inhalt streitig ist.

Die Klägerin mahnte den Beklagten zu 1) mit Schreiben vom 09. März 2011 und die Beklagte zu 2) mit Schreiben vom 15. Februar 2011 ab. Die Beklagte zu 2) gab daraufhin mit Schreiben ihrer Prozessbevollmächtigten vom 18. Februar 2011 eine Unterlassungserklärung ab. Die Klägerin nimmt den Beklagten zu 1) aus eigenem Recht und aufgrund des Schreibens der Senatsverwaltung für Stadtentwicklung und Umwelt vom 13. Dezember 2011 (Anlage K 15) als Prozessstandschafterin des Landes Berlin auf Unterlassung und beide Beklagten- die Beklagte zu 2) bis zur Abgabe der Erledigungserklärung - auf Auskunft hinsichtlich der Vervielfältigung und Verbreitung des Films „Unlike U“ in Anspruch.

Sie meint, ihr stünden die geltend gemachten Ansprüche aus $\S 823$ Abs. 1 BGB unter dem Gesichtspunkt eines Eingriffs in den eingerichteten und ausgeübten Gewerbebetrieb zu. Die ausführliche filmische Darstellung von zu ihren Lasten begangenen Sachbeschädigungen mit Musikuntermalung in der Art eines Musikvideos und Kommentierungen durch die Täter bestätige diese in ihrem Tun und fördere die Nachahmungsgefahr. Die Beklagten hätten damit die Beeinträchtigung ihres, i.e. des Betriebes der Klägerin, zumindest billigend in Kauf genommen. Ferner habe sie einen Anspruch aus $\S \S 823$ Abs. 2 BGB in Verbindung mit $\S \S 123,303$ StGB. Derjenige, der solche Aufnahmen im Einvernehmen mit den Tätern anfertige, leiste zu den darin gezeigten Straftaten der Sachbeschädigung und des Hausfrie-

Eingesandt von Dr. Benjamin Raue, Kiel. 
densbruchs psychische Beihilfe. Es bestehe der Anschein, dass die Aufnahmen im Auftrag oder zumindest mit Billigung der Regisseure erstellt worden seien.

Schließlich beruhten die Ansprüche auf $\S 1004$ BGB unter dem Gesichtspunkt einer Verletzung der dem Eigentümer kraft seines Eigentums ausschließlich zugewiesenen Verwertungsbefugnis. Die Grundsätze, die der BGH in seinen zu preußischen Gärten und Parkanlagen ergangenen Entscheidungen vom 17. Dezember 2010 (V ZR 44/10, V ZR 45/10, V ZR 46/10) aufgestellt habe, seien auf den hiesigen Sachverhalt übertragbar. Dazu behauptet die Klägerin, soweit durch die U-Bahnanlagen private Grundstücke Dritter in Anspruch genommen worden seien, bestünden hierfür beschränkte persönliche Dienstbarkeiten, wie es beispielhaft beim U-Bahnhof Mendelssohn-Bartholdy-Platz der Fall sei. Der weit überwiegende Teil der U-Bahnanlagen befinde sich im Land Berlin auf bzw. unter öffentlichem Straßenland oder sonstigen, im Eigentum des Landes Berlin befindlichen Flächen. Da das Land Berlin unstreitig ihr Gewährträger sei, bestehe für eine Sicherung der Grundstücksnutzung durch die Eintragung beschränkt persönlicher Dienstbarkeiten kein Bedürfnis. Zum Sondervermögen des Eigenbetriebs Berliner Verkehrsbetriebe, deren Gesamtrechtsnachfolgerin sie durch das Eigenbetriebsreformgesetz geworden sei, hätten sämtliche Personenbeförderungsanlagen und Beförderungsmittel gehört, insbesondere die Bahnhöfe einschließlich Zugangs- und Tunnelanlagen der Linien U 1 bis $\mathrm{U} 9$ sowie die U- Bahnfahrzeuge. Soweit die für ihren Betrieb in Anspruch genommenen Grundstücke nicht in ihrem Eigentum stünden, handele es sich, so meint die Klägerin, bei den U-Bahnanlagen um Scheinbestandteile im Sinne des § 95 BGB, die wegen ihrer, der Klägerin, Bindung an die Betriebsgenehmigungen nur zu einem vorübergehenden Zweck mit den Grundstücken verbunden worden seien. U-Bahnanlagen, die das Land Berlin für den damaligen Eigenbetrieb auf eigenen Grundstücken errichtet habe, seien durch das Eigenbetriebsreformgesetz auf sie, die Klägerin, übergegangen, wodurch sie die Eigenschaft als Grundstücksbestandteil verloren und den Status eines Scheinbestandteils angenommen hätten.

Sie, die Klägerin, habe der Anfertigung von Fotoaufnahmen innerhalb ihrer Betriebsanlagen nicht zugestimmt. Ihre Pressestelle sei auf das Anliegen des Herrn T. nur wegen des vermeintlich studentischen Hintergrunds eingegangen. Den wahren Charakter des Projekts habe Herr T. auch in der anschließenden Besprechung am 24. November 2009 nicht offenbart. Eine Erlaubnis zur Veröffentlichung der am 11. März 2010 erstellten Aufnahmen in einem beliebigen Zusammenhang habe weder Herr B. als technischer Hauptsachbearbeiter in der Betriebswerkstatt G., noch ihre Pressestelle erteilt.

Die Beklagte zu 2) hafte allein aufgrund des Vertriebs der Aufnahmen auf Schadenersatz, denn sie habe nicht nur eine Plattform für Angebote Dritter zur Verfügung gestellt, sondern selbst die Vermarktung des Films betrieben. Daher käme es nicht mehr darauf an, ob sie die Aufnahmen zusätzlich auch selbst hergestellt habe oder habe herstellen lassen.

Nachdem die Beklagte zu 2) die begehrte Auskunft in der Klageerwiderung erteilt hat, haben die Parteien den Rechtsstreit insoweit übereinstimmend für erledigt erklärt.

Die Klägerin beantragt jetzt noch,

den Beklagten zu 1) zu verurteilen,

1. es bei Meidung eines vom Gericht für jeden Fall der Zuwiderhandlung angedrohten Ordnungsgeldes bis zu 250.000 Euro, ersatzweise Ordnungshaft oder Ordnungshaft bis zu sechs Monaten, zu unterlassen, ungenehmigte Filmaufnahmen der Verkehrsmittel und / oder der Betriebsanlagen der Klägerin zu vervielfältigen / vervielfältigen zu lassen und / oder verbreiten / verbreiten zu lassen, soweit die Aufnahmen innerhalb der Betriebsanlagen und / oder Verkehrsmittel der Klägerin angefertigt wurden,

2. der Klägerin Auskunft darüber zu erteilen, in welchem Umfang sie die in Ziffer 1 genannten Handlungen in der Vergangenheit begangen haben, insbesondere unter Angabe der Anzahl der hergestellten und / oder veräußerten DVDs des Filmwerks „Unlike U - trainwriting in berlin" sowie der Angabe der Gesamtumsätze mit diesen DVDs einschließlich Kostenfaktoren und des Gewinns.

\section{Der Beklagte zu 1) beantragt,}

die Klage abzuweisen.

Er behauptet, sämtliche Aufnahmen illegaler Aktionen seien innen als Filmemacher anonym zugespielt worden. Die Klägerin, die ihren Wunsch nach einem aufgezeichneten Interview abgelehnt habe, habe sie für die beabsichtigte Herstellung von Aufnahmen über die Reinigung bemalter Züge an die Betriebswerkstatt Britz-Süd verwiesen. Da diese ihnen keinen Zeitpunkt für eine solche Reinigung habe benennen können, hätten sie sodann unstreitig die Reinigung eines unbemalten U-Bahnzuges in der Betriebswerkstatt Friedrichsfelde gefilmt. Bei dieser Gelegenheit hätten sie Herrn B. die Verwendung von Aufnahmen illegaler Sprühaktionen in ihrem Film offenbart. Im Übrigen habe Herr T. auch schon bei der Besprechung am 24. November 2009 darauf hingewiesen, dass die Aufnahmen auch für ein weiteres, nämlich sein, des Beklagten zu 1) Dokumentarfilmprojekt, hätten Verwendung finden sollen. Die Mitarbeiter der Klägerin hätten dagegen weder Einwände erhoben, noch Bedenken geäußert.

Der Beklagte zu 1) meint, für einen Anspruch aus $\S 823$ Abs. 1 BGB fehle es an einem betriebsbezogenen Eingriff. Ein Anspruch aus $\S 1004$ BGB wegen der Abbildung fremden Eigentums stehe der Klägerin selbst auf der Grundlage der genann- 
ten $B G H$-Rechtsprechung nicht zu. Dort habe sich der Wert der Aufnahmen darin erschöpft, einen bestimmten Gegenstand abzubilden. Der wirtschaftliche Nutzen sei allein aus dem Erscheinungsbild des Gegenstandes gezogen worden. Hier hingegen beruhe der Wert der Aufnahmen nicht darauf, dass darauf Züge und Bahnanlagen zu sehen seien, sondern darauf, dass es sich um Aufnahmen illegaler Aktionen handele. Der Nutzen, den sie, die Filmemacher, daraus zögen, beruhe allein darauf, diese Vorgänge, die sich üblicherweise im Verborgenen abspielen, jetzt für die Öffentlichkeit wahrnehmbar zu machen. Der erzielte Mehrwert sei journalistischer und nicht künstlerischer Natur. Zudem hätten die Täter die Aufnahmen nicht zum Zwecke der Verwertung angefertigt, sondern für ihren privaten Gebrauch, wie sich bereits aus dem Film selbst ergebe. Da er, der Beklagte zu 1), die Aufnahmen weder angefertigt, noch in Auftrag gegeben habe, hafte er nicht als (mittelbarer) Handlungsstörer. Die Klägerin halte zudem keine auf dem UrhG beruhenden Rechte an den abgebildeten Graffitis, bei denen es sich um schutzfähige Werke handele. Vielmehr hätten die jeweiligen Urheber ihnen, den Herstellern des Dokumentarfilms, die zur Auswertung des Films erforderlichen Nutzungsrechte uneingeschränkt eingeräumt. Der BGH habe in seiner genannten Rechtsprechung ausgeführt, dass Eigentümerbefugnisse insoweit eine Einschränkung erfahren könnten, als ihre Ausübung bestehende Urheberrechte verletze. Eben dies sei hier der Fall. Er, der Beklagte zu 1), bestreite zudem mit Nichtwissen, dass die gezeigten Bauwerke und sonstigen Örtlichkeiten, soweit sie Urheberschutz genössen, bereits gemeinfrei geworden seien. Die Verwendung der Aufnahmen sei durch die in Art. 5 GG garantierte Kunst- und Berichterstattungsfreiheit gerechtfertigt. Das Informationsinteresse der Öffentlichkeit überwiege die eigentumsrechtlichen Einbußen der Klägerin. Schließlich verhalte sich die Klägerin treuwidrig, weil sie bereits während der Produktion des Dokumentarfilms Kenntnis vom dem Vorhaben erlangt habe, ohne ihre Einwände zeitnah vorzubringen.

Die Beklagte zu 2) meint, sie hafte allenfalls als Störerin. Damit gehe kein Schadenersatzanspruch einher, der durch die Auskunft vorbereitet werden könne. Sie habe auch keine Eigentumsverletzung begangen, weil dies nach der o. g. Rechtsprechung des $B G H$ nicht nur die Verwertung, sondern kumulativ auch die Anfertigung der Aufnahmen voraussetze, die sie unstreitig nicht hergestellt habe.

\section{$[\ldots]$}

\section{Entscheidungsgründe}

Die Klage hat im jetzt noch geltend gemachten Umfang Erfolg.

1. Sie ist zulässig. Der Unterlassungsantrag genügt dem Erfordernis des § 253 ZPO. Er entspricht wörtlich der Formulierung des $B G H$ in der Entscheidung vom 17. Dezember 2010 (V ZR 46/10). Die Begriffe "Betriebsanlagen“ und "Verkehrsmittel" sind hinreichend bestimmt und aus sich heraus verständlich,
Unsicherheiten über Inhalt und Reichweite des Verbotes in Verbindung mit den ggfls. mit heranzuziehenden Entscheidungsgründen nicht erkennbar.

2. Der Klägerin steht der geltend gemachte Unterlassungsanspruch gegen den Beklagten zu 1) allerdings nicht schon aus $\S 823$ BGB unter dem Gesichtspunkt eines Eingriffs in den eingerichteten und ausgeübten Gewerbebetrieb zu, denn es fehlt an einem betriebsbezogenen Eingriff. Auch wenn durch dieses Recht im Grundsatz alles geschützt wird, was der unternehmerischen Betätigung und Entfaltung im Wirtschaftsleben dient, bedarf es zur Eingrenzung des Anspruchs der Betriebsbezogenheit des Eingriffs, der sich nach seiner objektiven Stoßrichtung gegen den betrieblichen Organismus oder die unternehmerische Entscheidungsfreiheit richten muss. Erforderlich ist eine Schadensgefahr, die geeignet ist, den Betrieb in empfindlicher Weise zu beeinträchtigen (BGH MMR 1998, 608 - Filmaufnahmen). Diese Voraussetzungen treffen zwar auf das Bemalen der U-Bahnzüge selbst zu, nicht aber auf das Verbreiten von Bildern derartiger Aktionen. Die Tatsache, dass die Aufnahmen erkennbar gegen den Willen der Klägerin zustande kamen, rechtfertigt für sich genommen nicht die Annahme eines rechtswidrigen Eingriffs, weil der Beklagte zu 1) insoweit das Grundrecht auf Kunstfreiheit für sich in Anspruch nehmen kann ( $B G H$ aaO für das Grundrecht aus Art. 5 Abs. 1 GG). Die gezeigten Straftaten sind nicht ihm, sondern den im Film gezeigten Personen zuzurechnen, die der Beklagte zu 1) auch nicht mit der Anfertigung der Aufnahmen beauftragt hat. Die in die gegenteilige Richtung weisenden Behauptungen der Klägerin entbehren jeder tatsächlichen Grundlage. Ob von den Bildern eine besondere Nachahmungsgefahr ausgeht, mag dahinstehen. Der Beklagte zu 1) ist dem Vorbringen der Klägerin zulässigerweise mit Nichtwissen entgegen getreten, ohne dass sie es mit Tatsachen, wie bspw. einem signifikanten Anstieg von Graffitibemalungen seit Erscheinen des Films untermauert hat. Selbst wenn man unterstellt, dass sich manche Zuschauer von dem Gezeigten zur Nachahmung anregen lassen könnten, beruht dies lediglich auf einem Reflex, der aus den anderweitig gegen den Betrieb der Klägerin gerichteten Eingriff erwächst. Ein solcher Reflex wäre in Abwägung mit dem widerstreitenden Grundrecht des Beklagten zu 1) auf Meinungs- und Kunstfreiheit hinzunehmen.

Der Anspruch folgt auch nicht aus §§ 823 Abs. 2 BGB in Verbindung mit $\S \S 123,303$ StGB. Zwar dürften das Bemalen der U-Bahnzüge und das Eindringen in die der Öffentlichkeit nicht zugänglichen Tunnelanlagen die Straftatbestände der Sachbeschädigung und des Hausfriedensbruchs verwirklichen. Der Beklagte zu 1) haftet dafür jedoch weder als Täter, weil er nicht vor Ort war und die Handlungen nicht selbst vornahm, noch als Teilnehmer. Beihilfe kann als Förderung der Haupttat nur bis zu deren Beendigung geleistet werden, die hier lange vor der Herstellung des Films eintrat. Das gleiche gilt hinsichtlich einer Anstiftung, die zudem voraussetzt, dass der Teilnehmer bei einem bestimmtem Haupttäter den Entschluss zu einer bestimmten Straftat weckt (Fischer, Strafgesetzbuch, 58. Aufl. 2011, Rn. 6 zu § 27 StGB und Rn. 6 ff zu § 26 StGB). Auch dies scheidet bei der 
nachträglichen Verbreitung der Filmaufnahmen aus, und zwar sowohl hinsichtlich der darin gezeigten Täter als auch hinsichtlich möglicher Nachahmer.

Der Klägerin steht der geltend gemachte Unterlassungsanspruch indes aus § 1004 Abs. 1 S. 2 BGB zu.

Der vom Beklagten zu 1) hergestellte Film verwertet Aufnahmen, die innerhalb der im Eigentum der Klägerin stehenden Betriebsanlagen erstellt wurden und deshalb ihr Grundstückseigentum verletzen.

Die im Hauptfilm bei etwa 00:32:33 beginnende Sequenz zeigt eine Gruppe Vermummter, die im U-Bahnhof OskarHelene-Heim einen Waggon bemalen und zum Schluss ihren Schriftzug „1 Up" auf dem Bahnsteig hinterlassen. Dabei wird der Bahnsteig nebst Aufbauten in voller Länge abgebildet. Das Bahnhofsschild ist deutlich zu erkennen, so dass Zweifel an der Örtlichkeit nicht aufkommen können. Dieselbe, etwas längere Sequenz findet sich im Bonus-Material im Titel "Subway", dort im Kapitel „1 Up Crew” mit reiner Musikuntermalung wieder. Eine weitere, im Hauptfilm etwa bei 01:25:57 beginnende Sequenz zeigt eine vermummte Person, die im U-Bahnhof Krumme Lanke in den Führerstand eines U-Bahn-Zuges eindringt, die Rückseite des Wagens während der Fahrt mit Farbe besprüht und die Gleise im U-Bahnhof Podbielskiallee, dessen Stationsschild deutlich erkennbar ist, wieder verlässt. Dieselbe Sequenz findet sich im Bonusmaterial im Titel "Subway”, dort im Kapitel „Fino" mit reiner Musikuntermalung wieder.

Die Aufnahmen wurden vom Bahnsteig bzw. aus der Fahrerkabine heraus „in Augenhöhe" mit den handelnden Personen und damit vom Grundeigentum der Klägerin aus angefertigt; denn diese hat durch die im Tatbestand aufgeführten Grundbuchauszüge substantiiert dargelegt, dass sie Eigentümerin der Grundstücke ist, auf denen sich die Bahnhöfe und der gezeigte Schienenstrang befinden. Damit stehen auch die Bahnhöfe selbst als wesentlicher Bestandteil des Grundstücks gemäß § 94 BGB im Eigentum der Klägerin. Sie ist zudem Eigentümerin der gezeigten U-Bahnwaggons. Dafür streitet die Vermutung des $\S$ 1006 BGB, die für alle Arten von Ansprüchen gilt, die Eigentum voraussetzen. Sie besagt, dass der Besitzer bei Besitzerwerb Eigenbesitz begründet, dabei unbedingtes Eigentum erwirbt und es während der Besitzzeit behält (Palandt-Bassenge BGB, 70. Aufl. 2010, § 1006 Rn. 4). Die Klägerin erwarb den Besitz an den Waggons spätestens in dem Moment, als sie auf das von ihr betriebene Schienennetz gesetzt wurden. Sofern dies noch zu Zeiten geschah, als die BVG als Eigenbetrieb des Landes Berlin geführt wurde, gilt § 1006 BGB in gleicher Weise zugunsten des Eigenbetriebs. Die Klägerin erlangte das Eigentum dann im Wege der Rechtsnachfolge durch die mit dem Eigenbetriebsreformgesetz einhergehende Umstrukturierung. Tatsachen, die diese Vermutung zu erschüttern vermögen, hat der Beklagte zu 1) nicht vorgetragen.
Nach der Rechtsprechung des $B G H$ (Urteil vom 17. Dezember 2010 - V ZR 46/10) stellen das ungenehmigte Filmen eines Gebäudes und die Verwertung der Bilder eine Eigentumsverletzung dar, wenn das Gebäude von dem Grundstück aus, auf dem es sich befindet, gefilmt wird, denn das Eigentum kann auch dadurch beeinträchtigt werden, dass es, ohne beschädigt zu werden, in einer dem Willen des Eigentümers widersprechenden Weise genutzt wird (aaO Tz. 17). Gemäß § 903 BGB bleibt dem Grundstückseigentümer die Entscheidung darüber vorbehalten, ob und in welchem Umfang er den Zugang zu seinem Grundstück eröffnet. Dies kann vollständig oder eingeschränkt geschehen. Hier gestattete die Klägerin den Zutritt zu ihren Anlagen unstreitig nur zur Inanspruchnahme der von ihr angebotenen Transportleistungen. Zu anderen Zwecken, wie bspw. Filmaufnahmen, gewährt sie den Zutritt nur im Einzelfall. Dass die Klägerin ihr Grundstück nicht für die filmische Dokumentation von strafbaren Handlungen im Moment ihrer Begehung zur Verfügung stellte, die sich noch dazu gegen ihr Eigentum richten, liegt auf der Hand und wird auch vom Beklagten zu 1) nicht ernstlich in Abrede gestellt.

Der Einwand des Beklagten zu 1), der Wert der Bilder verkörpere sich hier im Gegensatz zu dem vom BGH entschiedenen Sachverhalt nicht im Erscheinungsbild der abgebildeten Bahnanlagen, sondern in der Tatsache, dass sie illegale Sprühaktionen zeigen, überzeugt nicht. Der BGH hebt in seiner Argumentation nicht darauf ab, ob das Gebäude um seiner selbst willen oder aus anderen Gründen im Bild festgehalten wird. Darauf kommt es ebenso wenig an wie auf die Frage, ob der Eigentümer die Abbildungen in gleicher Art und Weise anfertigen oder verwerten könnte. Die Befugnis des Eigentümers, die Verwertung von Abbildungen seines Eigentums unter bestimmten Umständen unterbinden zu können, entspringt ausschließlich seiner absolut geschützten Rechtsposition als Eigentümer. Entscheidend ist allein, wo die im Streit stehenden Filmaufnahmen entstanden, nämlich auf dem Grundstück des Eigentümers oder außerhalb davon. Der Entscheidung des BGH kann daher auch nicht entnommen werden, dass es auf einen vom Beklagten zu 1) ins Feld geführten Kausalzusammenhang in dem Sinne ankäme, dass der Fotograf die Aufnahmen auf dem fremden Grundstück gerade zum Zwecke ihrer Verwertung anfertigen bzw. ihre kommerzielle Verwertung von Anfang an ins Auge fassen muss. Zwar heißt es in der o.g. Entscheidung (BGH V ZR 46/10) im Zusammenhang mit den Ausführungen zur Stellung des dortigen Beklagten als Handlungsstörer (Tz. 27), dass er bei den von ihm angebotenen DVDs über die Stadt Potsdam nicht auf fremdes Bild- und Filmmaterial zurückgegriffen habe. Gleichzeitig betont der BGH aber an früherer Stelle im Urteil (Tz. 17), dass die Beeinträchtigung des Eigentums durch die ungenehmigte Verwertung der ungenehmigten Abbilder noch vertieft würde. Daraus ist zu schließen, dass der Verwertung der auf dem Grundstück entstandenen Bildaufnahmen gegenüber ihrer Herstellung ein eigener - zusätzlicher - Unrechtsgehalt inne wohnt mit der Folge, dass auch die isolierte Verwertung fremder Bilder für sich genommen eine Eigentumsstörung darstellt, die der Eigentümer unabhängig davon unterbinden kann, 
ob der Verwerter das Eigentum zusätzlich auch noch durch die ungenehmigte Herstellung der Fotografien verletzte. Diese Sichtweise mag zwar insofern auf praktische Schwierigkeiten stoßen, als der Verwerter im Regelfall nicht weiß oder wissen kann, von welcher Position aus die Fotografien aufgenommen wurden und ob der Grundstückseigentümer der Herstellung der Bilder zugestimmt hat. Hier besteht aber die Besonderheit, dass den verwendeten Sequenzen schon aufgrund ihres Inhalts - Straftaten zu Lasten der Klägerin - auf der Stirn geschrieben stand, dass sie ohne ihre Zustimmung gedreht wurden. Das gilt erst recht bei zusätzlicher Berücksichtigung der Übergabemodalitäten durch anonyme Zuspielung aus der Szene. Die Verwertung dieser Bilder verletzt das Eigentum der Klägerin daher auch dann, wenn sie ursprünglich nur zu privaten Zwecken hergestellt wurden. Dass der Beklagte zu 1) die inm zugespielten Filmaufnahmen kommerziell verwertet, wenn er sie in einen Film integriert, den er u.a. zum Kauf auf DVD anbietet, kann keinem ernsthaften Zweifel unterliegen.

Die Klägerin hat der Verwertung der ungenehmigten Filmaufnahmen im Film „Unlike U” auch nicht zugestimmt. Soweit der Beklagte zu 1) geltend macht, er habe dem Mitarbeiter B. der Klägerin die Verwendung illegal hergestellter Filmaufnahmen offenbart und dieser habe nicht überrascht, irritiert oder ablehnend reagiert, lässt sich diesem Vorbringen eine Zustimmung des Herrn B. zur Verwendung der illegal hergestellten Filmaufnahmen nicht entnehmen. Zustimmung bedeutet mehr als fehlender Widerspruch, sie setzt ein aktives Signal des Einverständnisses voraus.

Der Beklagte zu 1) ist für den geltend gemachten Unterlassungsanspruch aus den genannten Gründen als Handlungsstörer passiv legitimiert, weil er mit der Verwertung der illegal entstandenen Filmaufnahmen im Film „Unlike U” das Eigentum der Klägerin selbst adäquat beeinträchtigte.

Die Klägerin muss die Verwertung der unerlaubt hergestellten Aufnahmen durch den Beklagten zu 1) auch nicht gemäß $\S$ 1004 Abs. 2 BGB dulden.

Eine solche Duldungspflicht ergibt sich nicht aus dem vom Beklagten zu 1) bemühten Urheberrecht. Dabei kann zu seinen Gunsten unterstellt werden, dass die Graffiti schutzfähige Werke der freien Kunst gemäß § 2 UrhG darstellen. Seine Behauptung, die Urheber hätten ihm die erforderlichen Nutzungs- und Verwertungsrechte daran eingeräumt, hat die Klägerin zulässigerweise mit Nichtwissen bestritten, ohne dass der Beklagte zu 1) seine Behauptung mit konkreten Tatsachen unterlegte. Eigene, aus dem UrhG abgeleitete (ausschließliche) Verwertungsrechte kann der Beklagte zu 1) daher nicht entgegensetzen. Der Rückgriff auf fremde Urheberrechte bleibt ihm versagt, weil er nicht Sachwalter der unbekannten Künstler ist.

Eine Pflicht, die Verwertung der Abbildungen ihres Eigentums dulden zu müssen, ergibt sich für die Klägerin auch nicht unter dem Gesichtspunkt der Kunstfreiheit. Art. 5 Abs. 3 GG erkennt ein individuelles Freiheitsrecht zu künstlerischer Betätigung an, das auch die Verbreitung von Kunstwerken einschließt. Die Vorschrift schützt vor Einwirkungen öffentlicher Gewalt auf Inhalt, Methoden und Tendenzen künstlerischer Tätigkeit. In diesem Bereich wird sie vorbehaltlos gewährt. Die Reichweite der Gewährleistung erstreckt sich aber von vornherein nicht auf die eigenmächtige Inanspruchnahme oder Beeinträchtigung fremden Eigentums (BVerfG NJW 1984, 1293). Dass die auf dem Eigentum der Klägerin angebrachten Graffitis durch die Untersagung der DVD „Unlike U” für die Öffentlichkeit nicht mehr wahrnehmbar sind, stellt daher keinen Eingriff in die Kunstfreiheit dar.

Für den Film, der für sich genommen dem verfassungsrechtlichen Kunstbegriff unterfällt, gilt im Ergebnis nichts anderes. Auch er macht sich die erkennbar eigenmächtige Inanspruchnahme fremden Eigentums zunutze, indem er auf der bildlichen Dokumentation dieser Handlungen aufbaut und diese unverändert und kommentarlos wiedergibt. Die Eigentumsgarantie, aus der die Klägerin ihren Unterlassungsanspruch ableitet, steht der Kunstfreiheit als ein anderes Freiheitsrecht gleichberechtigt gegenüber. Nach den vom GG getroffenen Wertungen tritt sie nicht prinzipiell hinter die Kunstfreiheit zurück (BVerfG aaO). Das gilt umso mehr, als es hier nur um die Modalitäten der Kunstausübung geht; denn dem Beklagten zu 1) ist es weder verboten, einen Film über Graffitis im öffentlichen Nahverkehr und die dazugehörige Szene herzustellen und der Öffentlichkeit zu präsentieren, noch, Bilder von bemalten U-Bahnzügen zu zeigen. Er kann solche Aufnahmen weiterhin verwenden, sofern sie an Standorten außerhalb des Eigentums der Klägerin entstanden, wie es bspw. bei den Bildern der Fall ist, die in der eingangs beschriebenen Sequenz von erhöhter Warte aus auf Überführungen der Bahngleise gedreht wurden.

Schließlich kann der Beklagte zu 1) aus denselben Gründen auch nicht mit Erfolg auf das Grundrecht der Pressefreiheit (Art. 5 Abs. 1 S. 2 GG) verweisen. In der Abwägung der sich gegenüber stehenden Grundrechtspositionen überwiegt das Interesse des Beklagten zu 1) daran, die Öffentlichkeit mittels illegal hergestellter Bildaufnahmen über das Problem des Graffitis speziell auf den von der Klägerin benutzten Zügen zu informieren, nicht das oben geschilderte, dem Eigentum der Klägerin entspringende Interesse, ihr Eigentum für solche Verwertungen nicht zur Verfügung zu stellen. Dabei ist auf Seiten des Beklagten zu 1) zu berücksichtigen, dass die Darstellung der Straftat in bewegten Bildern dem Zuschauer zwar eine besondere Authentizität des Geschehens vermittelt, der damit einhergehende Erkenntnisgewinn gegenüber bspw. einer mündlichen Schilderung der Vorgänge oder der Einblendung von Standfotos indes eher gering bleibt. Die beanstandete Sequenz dient damit vornehmlich der Befriedigung der Neugierde des Zuschauers, vermittelt ihm aber keine weitergehenden Informationen. Dass die Klägerin dieser Vorgehensweise des Beklagten zu 1) durch die Verwertung von Abbildungen ihres Eigentums nicht Vorschub leisten möchte, ist ein anerkennenswertes Motiv für die Geltendmachung ihrer Eigentumsrechte. 
3. Der Klägerin steht gegen den Beklagten zu 1) ferner gemäß § 242 in Verbindung mit $\S 823$ BGB ein Auskunftsanspruch im geltend gemachten Umfang zu. Der $B G H$ hat die Rechtsprechung, die bei Urheberrechtsverletzungen dem Betroffenen zur Vorbereitung eines bezifferten Schadenersatzanspruches eine auf Treu und Glauben beruhende Auskunftspflicht auferlegt, sofern der Verletzte in entschuldbarer Weise über das Bestehen oder den Umfang seines Anspruchs auf Schadenersatz oder Bereicherung im Unklaren ist, auf die Beeinträchtigung des Eigentümers in seiner Befugnis zur Verwertung des Erscheinungsbildes der Sache übertragen (BGH aaO Tz. 34). Der Beklagte zu 1) handelte bei der Verwertung der ihm zugespielten Filmaufnahmen ohne Beachtung der im Verkehr erforderlichen Sorgfalt und damit schuldhaft. Zwar erging die zitierte Rechtsprechung des BGH erst im Dezember 2010 und damit zu einer Zeit, in der die DVD „Unlike U” möglicherweise bereits vertrieben wurde. Die erkennbare Abbildung von Straftaten hätte beim Beklagten zu 1) jedoch auch ohne Kenntnis dieser Rechtsprechung Zweifel daran wecken müssen, ob er die Aufnahmen ohne Zustimmung der Klägerin, die Opfer der Straftaten wurde, kommerziell verwerten darf. Rechtsrat dazu hat er nicht eingeholt.

4. Die Kostenentscheidung beruht unter Berücksichtigung der in Bezug auf den Streitwert und die Verfahrensstufen unterschiedlichen Beteiligung beider Beklagten auf §§ 91, 91a ZPO. Soweit die Klägerin den gegen die Beklagte zu 2) gerichteten Auskunftsanspruch in der Hauptsache für erledigt erklärt und sich die Beklagte zu 2) der Erledigungserklärung angeschlossen hat, hat die Beklagte zu 2) den darauf entfallenden Kostenanteil zu tragen. Die Klage hätte voraussichtlich Erfolg gehabt, weil die Beklagte zu 2) den Film selbst durch den Verkauf der DVDs verbreitete und damit die Tathandlung selbst als Täterin beging. Nach herkömmlicher Ansicht ist Täter, wer willentlich eine Handlung vornimmt, die den Tatbestand verwirklicht. Die Rechtswidrigkeit seines Tuns muss dem Handelnden dabei nicht bewusst sein (Jan Bernd Nordemann in Fromm/Nordemann, Urheberrecht, 10. Rdnr. 150 zu § 97; Wild in Schricker/ Loewenheim, 4. Aufl., Rdnr. 122 zu § 97, jeweils mwN). Ebensowenig kommt es für die Einordnung einer Verhaltensweise als täterschaftliches Handeln auf Verschuldensgesichtspunkte an. Diese gewinnen im Rahmen der hier begehrten Auskunft erst dadurch Bedeutung, dass ein Schadenersatzanspruch, dessen Vorbereitung die Auskunft dienen soll, Verschulden voraussetzt. Ob die Beklagte zu 2) ein Verschuldensvorwurf trifft, kann hier gleichwohl offen bleiben, weil die Klägerin die Auskunft in Übereinstimmung mit der o.a. Rechtsprechung des $B G H$ (aaO Tz. 34) erklärtermaßen auch zur Berechnung eines verschuldensunabhängigen Bereicherungsausgleichs begehrt. Allerdings mehren sich in Rechtsprechung und Literatur Stimmen, die eine täterschaftliche Haftung für "technische Multiplikatoren" wie bspw. Online-Buchhändler in Frage stellen (LG Berlin, 15 O 120/08 NJW 2009, 787 = GRUR-RR 2009, 216; LG Hamburg, 308 O 16/11 = GRUR-RR 2011, 249; LG Düsseldorf, 12 O 5/09 = ZUM-RD 2009, 279). Teils wird dies mit fehlender Tatherrschaft (so LG Berlin aaO), teils mit dem verfassungsrechtlichen Schutz der Medienfreiheit (so LG Hamburg aaO) begründet. Es kann dahingestellt bleiben, ob diesen Ansichten generell zu folgen ist, da hier eine andere Sachverhaltskonstellation vorliegt. Den genannten Entscheidungen ist gemeinsam, dass die dort beanstandeten Werke nur jeweils eines von vielen bildeten, die der jeweilige Online-Buchhändler auf seiner Internetseite zum Erwerb anbot. Hier mag zwar die Beklagte zu 2) insgesamt über ein umfangreiches Gesamtsortiment verfügen; die beanstandete DVD bot sie indes nicht auf einer Internetseite an, die auf ihr Gesamtangebot verweist, sondern zusammen mit dazu passenden Merchandising-Artikeln auf einer speziell eingerichteten Internetseite, die den Titel des Films bereits in der Domain www. xxx.com führt. Sie hebt dadurch gerade dieses Werk aus der Masse sonstiger Filme heraus und verlässt damit die Rolle eines reinen - und damit Distanz wahrenden - "Vertriebswerkzeugs" des Produzenten. Stattdessen tritt sie dem Verkehr als eine Anbieterin gegenüber, die dem Produkt breiten Raum bietet und damit zum Ausdruck bringt, dass sie in besonderer Weise "hinter dem Produkt steht", sei es, weil sie sein Thema für besonders wichtig erachtet, sei es, weil sie die Qualität des Werkes schätzt. Unter diesen Umständen und gerade auch im Hinblick auf das ergänzende Merchandising-Angebot kann nicht mehr von einem fehlenden Tatherrschaftswillen ausgegangen werden, dies umso weniger, als bereits das Cover der DVD auf der Rückseite am Schluss der Inhaltsbeschreibung mit der Formulierung „Extrem kriminell. Extrem verboten" auf die Wiedergabe von Straftaten im Film hinweist.

$\mathrm{Da}$, wie oben ausgeführt, das Verbreiten des Films unabhängig von seiner Herstellung als eigene Verletzungshandlung anzusehen ist, die Beklagte zu 2) diese Handlung als Täterin beging und dadurch unerlaubt in den der Klägerin vorbehaltenen Zuweisungsgehalt des Eigentums eingriff, schuldet sie Herausgabe des Erlangten und zur Vorbereitung dieses Anspruchs die erteilte Auskunft. [...]. 